\title{
The Microscopy of Materials for Interfacing Bionic Devices with Humans
}

\author{
David C. Martin
}

Karl W. and Renate Böer Professor and Chair, Materials Science and Engineering, The University of Delaware, Newark DE 19716

There is considerable interest in the design, development, and optimization of materials for interfacing hard, metallic, electrically conducting biomedical devices with soft, ionically conducting, organic biological tissue. Examples of these devices include cochlear implants, microfabricated cortical electrodes, deep brain stimulators, and pacemakers.

Our research group has been developing organic conducting polymers that provide significant advantages over existing materials at bionic interfaces. These films can be electrochemically deposited directly onto a metal electrode using oxidative electrochemical deposition techniques [1]. They significantly lower the impedance of both stimulating and recording electrodes in the biologically-relevant $1 \mathrm{kHz}$ frequency range [2]. They can also be used to locally deliver neurotrophic factors [3] or anti-inflammatory agents [4]. Most recently, we have focused on the use of in-situ techniques for the direct polymerization of these materials around living cells both in-vitro [5] and in-vivo [6].

In our studies we use an extensive compliment of techniques to determine the detailed morphology of our materials including polarized transmitted and reflected optical microscopy, confocal optical microscopy, scanning electron microscopy, scanned probe microscopy, transmission electron microscopy, scanning transmission electron microscopy, and low voltage electron microscopy. Figure 1 is a cross-section of a microfabricated neural prosthetic electrode coated with poly $(3,4-$ ethylene dioxythiophene) (PEDOT), obtained with an FEI Helios NanoLab 600 dual beam FIB/SEM system.

\section{References}

[1] X. Cui et al., Sens. Actuat. A, 93, (2001), 8.

[2] X. Cui and D. Martin, Sens. Actuat. B, 89 (2003).

[3] M. Abidian et al., Adv. Matls., 18 (2006) 405.

[4] M. Abidian and D. Martin, Adv. Func. Matls., 19 (2009) 573.

[5] S. Richardson-Burns, Biomaterials, 28, (2007), 1539.

[6] S. Richardson-Burns, J. Neural Eng., 4 (2007), L6.

[4] This research was supported by the National Science Foundation, the National Institute of Health, the Keck Futures Initiative and a U. S. Army MURI on Bio-Integrating Structural and Neural Prosthetic Materials. DCM is a Co-Founder and Chief Scientific Officer for Biotectix LLC, an Ann Arbor, MI based company actively working to commercialize the use of conducting polymer materials for a variety of biomedical device applications. Thanks are due to FEI in Hillsboro, OR for providing access to and assistance with FIB/SEM, and to Dr. Bong Sup Shim for sample preparation. 


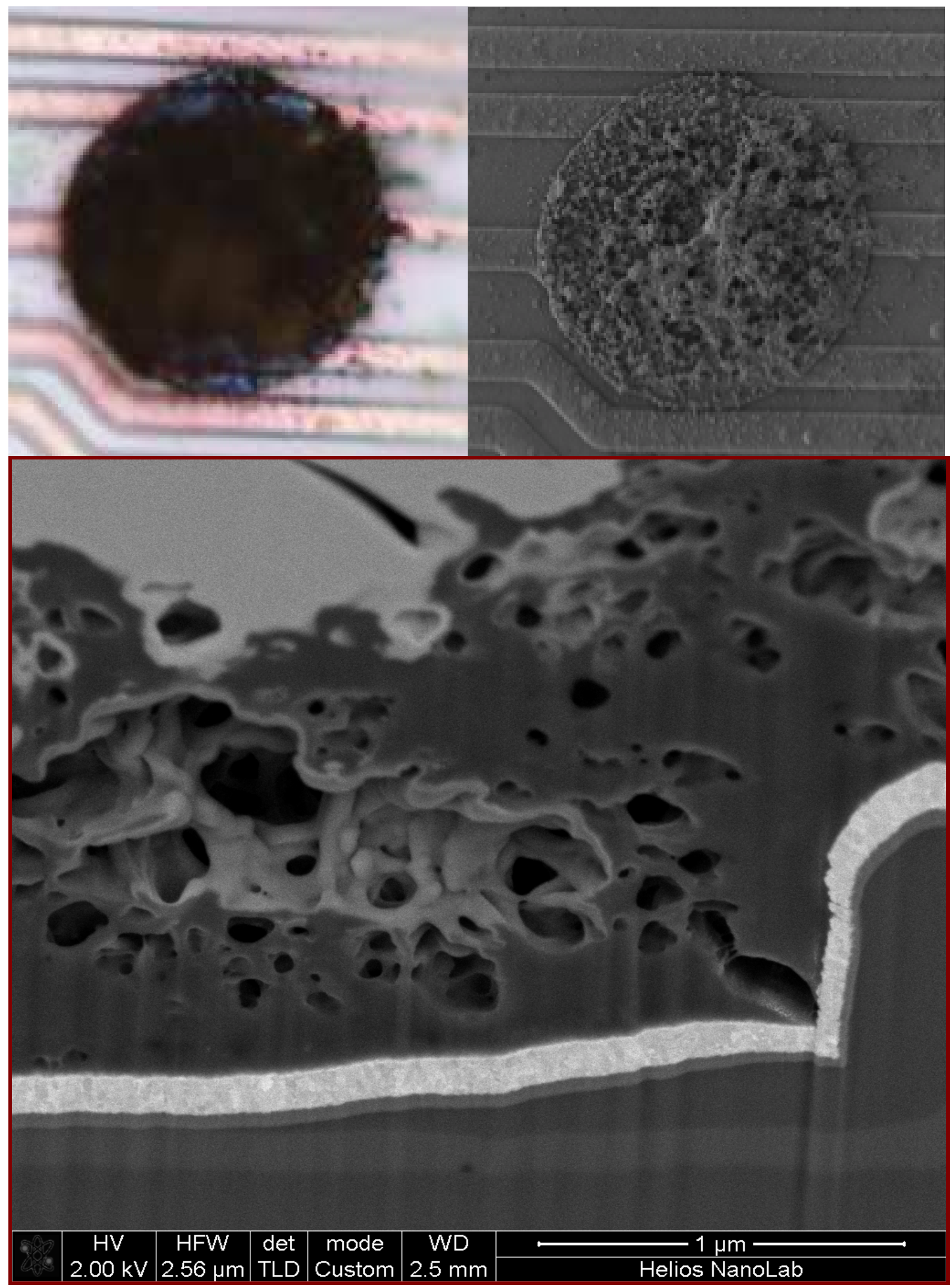

FIG. 1. Top L: Optical micrograph of a fuzzy PEDOT coating on a 40 micron microfabricated electrode. Top R: SEM of same fuzzy PEDOT coating. Bottom: FIB cross section of the PEDOT-metal electrode interface. 